\title{
Frobenius Algebras in Tensor Categories and Bimodule Extensions
}

\author{
Shigeru Yamagami \\ Department of Mathematical Sciences \\ Ibaraki University \\ Mito, 310-8512, JAPAN
}

\section{Introduction}

By recent research developments, the notion of tensor category has been recognized as a fundamental language in describing quantum symmetry, which can replace the traditional method of groups for investigating symmetry.

The terminology of tensor category is used here as a synonym of linear monoidal category and hence it has a good affinity with semigroup. One way to incorporate the invertibility axiom of groups is to impose rigidity (or duality) on tensor categories, which will be our main standpoint in what follows.

When a tensor category bears a finite group symmetry inside, it is an interesting problem to produce a new tensor category by taking quotients with respect to this inner symmetry. For quantum symmetries of rational conformal field theory, this kind of constructions are worked out in a direct and individual way with respect to finite cyclic groups.

In our previous works, these specific constructions are organized by interpreting them as bimodule tensor categories for the symmetry of finite groups with a satisfactory duality on bimodule extensions [12]. The construction is afterward generalized to the symmetry of tensor categories governed by finite-dimensional Hopf algebras [13].

We shall present in this paper a further generalization to symmetries described by categorical Frobenius algebras, which are formulated and utilized by J. Fuchs and C. Schweigert for a mathematical description of boundary conditions in conformal field theory [3] (see [5] for earlier studies on categorical Frobenius structures). A similar notion has been introduced under the name of Q-systems by R. Longo in connection with subfactory theory ([6], cf. also [9]). More precisely, a Q-system, if it is algebraically formulated, is equivalent to giving a Frobenius algebra satisfying a certain splitting condition, which is referred to as a special Frobenius algebra according to the terminology in [3].

(C)0000 American Mathematical Society

1991 Mathematics Subject Classification. 18D10, 46L37.

Key words and phrases. Frobenius algebra, tensor category, bimodule.

The first was supported in part by NSF Grant \#000000. 
Since our viewpoint here is that Q-systems (or special Frobenius algebras) should play the role of group algebras in classical symmetries, we first give an autonomic status to categorical Frobenius algebras as algebraic systems, which enables us to introduce the dual Frobenius algebras without assuming background tensor categories, together with a satisfactory duality on Frobenius algebras.

On the other hand, if Frobenius algebras are realized inside a tensor category $\mathcal{T}$, it is fundamental to consider bimodule extensions of $\mathcal{T}$ and we shall generalize the duality result on bimodule extensions to symmetries specified by categorical Frobenius algebras.

More precisely, given a special Frobenius algebra $A$ realized inside a tensor category $\mathcal{T}$, we show the existence of a natural imbedding of the dual Frobenius algebra $B$ of $A$ into the tensor category ${ }_{A} \mathcal{T}_{A}$ of $A$ - $A$ bimodules in $\mathcal{T}$. The duality for bimodule extensions is then formulated so that the second bimodule extension ${ }_{B}\left({ }_{A} \mathcal{T}_{A}\right)_{B}$ of $B-B$ bimodules in ${ }_{A} \mathcal{T}_{A}$ is naturally isomorphic (monoidally equivalent) to the starting tensor category $\mathcal{T}$.

The author is greatful to A. Masuoka and M. Müger for helpful communications on the subject during the preparation of this article.

Convention: By a tensor category over a field $\mathbb{K}$, we shall mean a $\mathbb{K}$-linear category together with a compatible monoidal structure. If semisimplicty is involved, we assume that $\mathbb{K}$ is an algebraically closed field of zero characteristic.

Since we are primarily interested in the use for quantum symmetry, we shall not discriminate tensor categories as long as they provide the equivalent information; we shall implicitly assume the strictness of associativity as well as the saturation under taking direct sums and subobjects for example.

For basic categorical definitions, we refer to the standard text [8].

\section{Monoidal Algebras}

Let $\mathcal{T}$ be a strict tensor category over a field $\mathbb{K}$ and assume that $\operatorname{End}(I)=\mathbb{K} 1_{I}$ for the unit object $I$. Given an object $X$ in $\mathcal{T}$, set

$$
A_{m, n}=\operatorname{Hom}\left(X^{\otimes n}, X^{\otimes m}\right)
$$

for non-negative integers $m, n$. The family $\left\{A_{m, n}\right\}_{m, n \geq 0}$ is then a block system of algebra in the sense that $A=\oplus_{m, n \geq 0} A_{m, n}$ is an algebra satisfying $A_{k, l} A_{m, n} \subset$ $\delta_{l, m} A_{k, n}$ and $A_{0,0}=\mathbb{K}$. Denote the unit of $A_{n}$ by $1_{n}$.

The tensor product in the category $\mathcal{T}$ defines a bilinear map

$$
A_{k, l} \times A_{m, n} \ni f \times g \mapsto f \otimes g \in A_{k+m, l+n}
$$

such that

1. the unit $1_{0}$ of $A_{0}$ satisfies $1_{0} \otimes f=f \otimes 1_{0}=f$,

2. the tensor product is associative; $(f \otimes g) \otimes h=f \otimes(g \otimes h)$ and

3. compatible with the composition; $(f \otimes g)\left(f^{\prime} \otimes g^{\prime}\right)=\left(f f^{\prime}\right) \otimes\left(g g^{\prime}\right)$.

A block system of algebra is called a monoidal algebra according to Kazhdan and Wenzl [4] (though they use this terminology in a more restricted meaning) if it is furnished with the operation of taking tensor products which satisfies the above conditions.

Conversely, given a monoidal algebra $A$, we define a tensor category $\mathcal{A}$ in the following way; objects in $\mathcal{A}$ are parametrized by non-negative integers and the homset $\operatorname{Hom}(m, n)$ is the vector space $A_{n, m}$ with the composition of morphisms given 
by the multiplication in the algebra $A$. The tensor product operation in $\mathcal{A}$ is the one naturally induced from that of monoidal algebra. If the monoidal algebra $A$ is associated to an object $X$ in a tensor category $\mathcal{T}$, the tensor category $\mathcal{A}$ associated to $A$ is monoidally equivalent to the tensor category generated by $X$.

If the starting tensor category is semisimple, the monoidal algebra is locally semisimple in the sense that for any finite subset $F$ of non-negative integers, the subalgebra $\oplus_{i, j \in F} A_{i, j}$ is semisimple. Conversely, a locally semisimple monoidal algebra $A$ gives rise to a semisimple tensor category $\overline{\mathcal{A}}$ as the Karoubian envelope of $\mathcal{A}$ : an object in $\overline{\mathcal{A}}$ is a pair $(n, e)$ of an integer $n \geq 0$ and an idempotent $e$ in $A_{n}$ with hom-sets defined by

$$
\operatorname{Hom}((m, e),(n, f))=f A_{n, m} e .
$$

The operation of tensor product is given by

$$
(m, e) \otimes(n, f)=(m+n, e \otimes f)
$$

on objects.

A similar construction works for bicategories as well; consider a (strict) bicategory of two objects $\{1,2\}$ for example and choose objects $X, Y$ in the hom-categories $\operatorname{Hom}(2,1), \mathcal{H o m}(1,2)$ respectively. By using the tensor product notation for the composition in the bicategory, we have the four systems of block algebras

$$
\begin{aligned}
A_{m, n} & =\operatorname{Hom}\left((X \otimes Y)^{\otimes n},(X \otimes Y)^{\otimes m}\right), \\
B_{m+1, n+1} & =\operatorname{Hom}\left((X \otimes Y)^{\otimes n} \otimes X,(X \otimes Y)^{\otimes m} \otimes X\right), \\
C_{m+1, n+1} & =\operatorname{Hom}\left(Y \otimes(X \otimes Y)^{\otimes n}, Y \otimes(X \otimes Y)^{\otimes m}\right), \\
D_{m, n} & =\operatorname{Hom}\left((Y \otimes X)^{\otimes n},(Y \otimes X)^{\otimes m}\right)
\end{aligned}
$$

(note that $(X \otimes Y)^{\otimes n} \otimes X=X \otimes(Y \otimes X)^{\otimes n}$ are alternating tensor products of $X$ and $Y$ ) with the operation of tensor product among them applied in a $2 \times 2$-matrix way,

$$
\begin{aligned}
& A_{m, m^{\prime}} \otimes B_{n, n^{\prime}} \subset B_{m+n, m^{\prime \prime}+n^{\prime \prime},}, \\
& B_{m, m^{\prime}} \otimes D_{n, n^{\prime}} \subset B_{m+n, m^{\prime}+n^{\prime}}, \\
& C_{m, m^{\prime}} \otimes A_{n, n^{\prime}} \subset C_{m+n, m^{\prime}+n^{\prime}}, \\
& D_{m, m^{\prime}} \otimes C_{n, n^{\prime}} \subset C_{m+n, m^{\prime}+n^{\prime}}, \\
& B_{m, m^{\prime}} \otimes C_{n, n^{\prime}} \subset A_{m+n-1, m^{\prime}+n^{\prime}-1}, \\
& C_{m, m^{\prime}} \otimes B_{n, n^{\prime}} \subset D_{m+n-1, m^{\prime}+n^{\prime}-1},
\end{aligned}
$$

which satisfies the associativity and multiplicativity (and the unit condition for tensor products involving $A_{0}$ or $D_{0}$ ) exactly as in the definition of monoidal algebra.

Conversely, given such an algebraic system, we can recover a (two-object) bicategory together with off-diagonal objects $X$ and $Y$ in an obvious way.

We can also talk about isomorphisms of monoidal algebras or their bicategorical counterparts, which exactly correspond to isomorphisms between associated tensor categories or bicategories.

\section{Frobenius Algebras}

It would be just a formal business to formulate axioms of algebraic systems in terms of categorical languages such as monoids or algebras, see [8] for example. Here is a bit more elaborate formulation of Frobenius algebra structure in tensor 
categories, which we shall describe here, following [3] and [9], mainly to fix the notation with some rewritings of axioms.

Let $\mathcal{T}$ be a tensor category. An algebra in $\mathcal{T}$ is a triplet $(A, T, \delta)$ with $A$ an object in $\mathcal{T}, T \in \operatorname{Hom}(A \otimes A, A)$ and $\delta \in \operatorname{Hom}(I, A)$ satisfying $T\left(T \otimes 1_{A}\right)=$ $T\left(1_{A} \otimes T\right), T\left(\delta \otimes 1_{A}\right)=1_{A}=T\left(1_{A} \otimes \delta\right)$, which are graphically denoted in the following way:
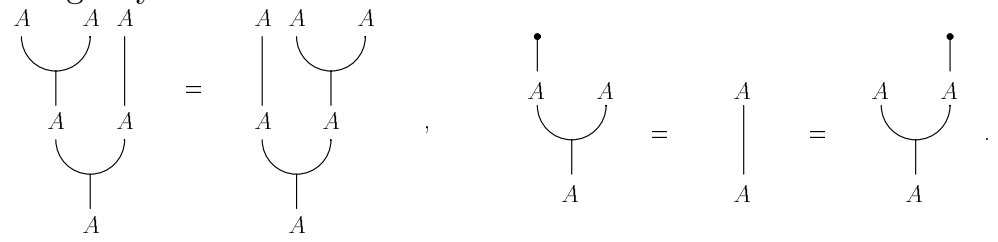

By reversing the direction of arrows, a coalgebra in $\mathcal{T}$ is a triplet $(C, S, \epsilon)$ with $S: C \rightarrow C \otimes C$ and $\epsilon: C \rightarrow I$ satisfying $\left(S \otimes 1_{C}\right) S=\left(1_{C} \otimes S\right) S,\left(\epsilon \otimes 1_{C}\right) S=1_{C}=$ $\left(1_{C} \otimes \epsilon\right) S:$<smiles>CCC(C)CCC(C)CCC(CC)CC</smiles><smiles>CCC(C)CC(C)CC(C)CC</smiles>

Note that $\delta$ and $\epsilon$ are uniquely determined by $T$ and $S$ respectively.

A Frobenius algebra in $\mathcal{T}$ is, by definition, a quintuplet $(A, S, T, \delta, \epsilon)$ with $(A, T, \delta)$ an algebra and $(A, S, \epsilon)$ a coalgebra, which satisfies the compatibility condition (st-duality), Fig. 1. The terminology is justified because the axioms turn out to be equivalent to those for ordinary Frobenius algebras if we work with the tensor category of finite-dimensional vector spaces. For an early appearance of categorical Frobenius structures, see [5].
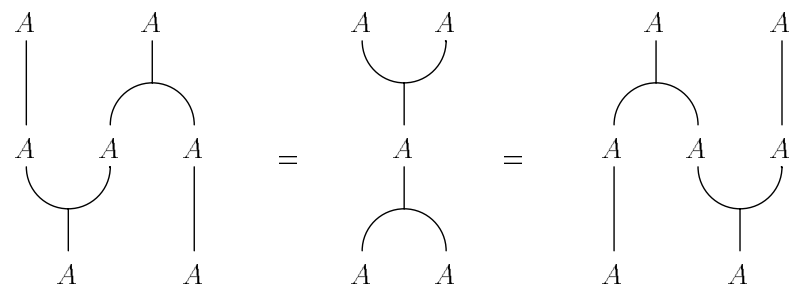

Figure 1

For a Frobenius algebra $(A, S, T, \delta, \epsilon)$, the object $A$ is self-dual with the rigidity pair given by

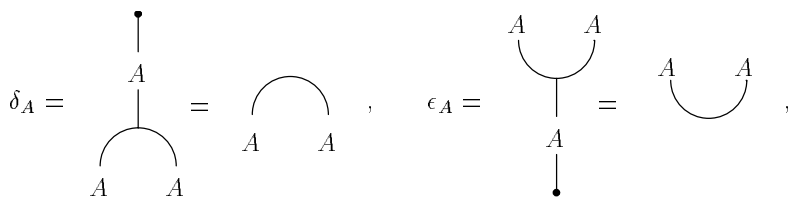

which satisfies the conditions 


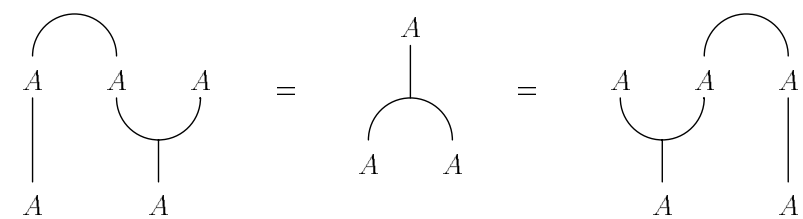

and

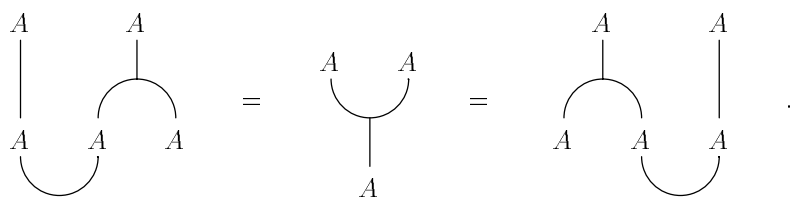

Conversely, given an algebra $(A, T, \delta)$ in $\mathcal{T}$ with $A$ a self-dual object and a rigidity copairing $\delta_{A}: I \rightarrow A \otimes A$ fulfilling the compatibility condition

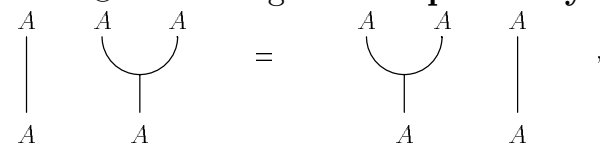

we can recover the Frobenius algebra by straightforward arguments so that the above common morphism $A \rightarrow A \otimes A$ serves as comultiplication. Since algebra and coalgebra structures are interchangeable with each other in the present context, we have the following characterizations of Frobenius algebra.

Proposition 2.1 Let $A$ be an object in a tensor category $\mathcal{T}$. Then the following data give the equivalent information on $A$.

1. A Frobenius algebra structure on $A$.

2. An algebra structure $(T, \delta)$ on $A$ together with a rigidity copairing $\delta_{A}: I \rightarrow$ $A \otimes A$ satisfying the compatibility condition ( $A$ being self-dual particularly).

3. A coalgebra structure $(S, \epsilon)$ on $A$ together with a rigidity pairing $\epsilon_{A}: A \otimes A \rightarrow$ I satisfying the compatibility condition.

4. A pair of morphisms $(S, T)$ satisfying the st-duality and the existence of units and counits.

It would be worth pointing out here that, in a $\mathrm{C}^{*}$-tensor category $\mathcal{T}$, any coalgebra $(A, S, \epsilon)$ is canonically supplemented to a Frobenius algebra (with the coalgebra structure given by taking adjoints of $S$ and $\epsilon$ ) provided that $S$ is a scalar multiple of an isometry [7].

In what follows, we shall assume that

$$
T S=(\text { non-zero scalar }) 1_{A} \quad \text { and } \quad \epsilon \delta=(\text { non-zero scalar }) 1_{I} .
$$

Note that the st-duality relation for the pair $(S, T)$ uniquely determines $\epsilon$ and $\delta$. For example, if we change $(S, T)$ into $(\lambda S, \mu T)$, then $(\epsilon, \delta)$ is modified into $\left(\mu^{-1} \epsilon, \lambda^{-1} \delta\right)$. Thus, by adjusting scalar multiplications, we may assume that the scalars appearing in $T S$ and $\epsilon \delta$ coincide. If this is the case, we call the pair $(S, T)$ an algebraic Qsystem (see [6] for the original meaning of Q-systems) and denote the common scalar by $d$. The associated Frobenius algebra is then referred to as a special Frobenius algebra according to [3]. (In [9], the adjective 'strongly separable' is used instead of 'special'.)

A standard model for special Frobenius algebras is the following: Assume that we are given a (strict) bicategory of two objects $\{1,2\}$ and arrange the associated four hom-categories in the matrix form $\left(\begin{array}{ll}\mathcal{H}_{11} & \mathcal{H}_{12} \\ \mathcal{H}_{21} & \mathcal{H}_{22}\end{array}\right)$ with $\mathcal{H}_{i j}=\mathcal{H o m}(j, i)$. 
Choose off-diagonal objects $H \in \mathcal{H}_{12}$ and $H^{*} \in \mathcal{H}_{21}$ such that $H^{*}$ is a left and right dual of $H$ at the same time with a (right) rigidity pairing $\epsilon: H^{*} \otimes H \rightarrow I_{2}$ and a (left) copairing $\delta: I_{2} \rightarrow H^{*} \otimes H$. Then $A=H \otimes H^{*}$ is a Frobenius algebra with multiplication and comultiplication given by $T=1_{H} \otimes \epsilon \otimes 1_{H^{*}}$ and $S=1_{H} \otimes \delta \otimes 1_{H^{*}}$ respectively.

If we further assume the irreducibility of $H$ as well as the existence of unit objects $I_{1} \in \mathcal{H}_{11}$ and $I_{2} \in \mathcal{H}_{22}$, then $A$ is a special Frobenius algebra.

\section{Remark 2.2}

1. If we consider the case of the tensor category of normal *-endomorphisms of an infinite factor, we are reduced to the situation of Q-systems in [6], [7].

2. See [9] for more information on the relationship with the notion of Q-system.

\section{Dual Systems}

Given an algebraic Q-system and objects $X, Y$ in $\mathcal{T}$, we introduce an idempotent operator $E=E_{Y, X}: \operatorname{Hom}(A \otimes X, A \otimes Y) \rightarrow \operatorname{Hom}(A \otimes X, A \otimes Y)$ by

$$
E(f)=\frac{1}{d}\left(T \otimes 1_{Y}\right)\left(1_{A} \otimes f\right)\left(S \otimes 1_{X}\right),
$$

where $d$ is the non-zero scalar associated to the algebraic Q-system.

The following is an easy consequence of graphical computations.

Lemma 3.1 For $f \in \operatorname{Hom}(A \otimes X, A \otimes Y)$, the following conditions are equivalent.

1. $E(f)=f$.

2. $f\left(T \otimes 1_{X}\right)=\left(T \otimes 1_{Y}\right)\left(1_{A} \otimes f\right)$.

3. $\left(S \otimes 1_{Y}\right) f=\left(1_{A} \otimes f\right)\left(S \otimes 1_{X}\right)$.

Corollary 3.2 The image of $\operatorname{End}(A \otimes X)$ under the map $E$, i.e., $\{f \in \operatorname{End}(A \otimes$ $X) ; E(f)=f\}$, is a subalgebra of $\operatorname{End}(A \otimes X)$.

Similarly we can introduce the idempotent operator $F$ associated to the right tensoring of $A$. We consider the monoidal algebra $\left\{A_{m, n}=\operatorname{Hom}\left(A^{\otimes n}, A^{\otimes m}\right)\right\}_{m, n \geq 0}$ associated with the object $A$. Set

$$
D_{m, n}=\left\{f \in A_{m+1, n+1} ; E(f)=f \text { and } F(f)=f\right\}
$$

for $m, n \geq 0$ and

$$
\begin{aligned}
& B_{m, n}=\left\{f \in A_{m, n} ; F(f)=f\right\}, \\
& C_{m, n}=\left\{f \in A_{m, n} ; E(f)=f\right\}
\end{aligned}
$$

for $m, n \geq 1$. Note here that $E F=F E$ on $A_{m+1, n+1}$ by the associativity of $S$ and $T$.

The above corollary then shows that $\left\{D_{m, n}\right\}_{m, n \geq 0}$ is a block system of algebra, i.e., $D_{k, l} D_{m, n} \subset \delta_{l, m} D_{k, n}$, where the product is performed inside the block system of algebra $\bigoplus_{i, j \geq 0} A_{i, j}$. Similarly for $\left\{B_{m, n}\right\}_{m, n \geq 1}$ and $\left\{C_{m, n}\right\}_{m, n \geq 1}$.

We shall now make $\left\{D_{k, l}\right\}$ into a monoidal algebra. Let $f \in D_{k, l}$ and $g \in D_{m, n}$. We define $f \widehat{\otimes} g \in A_{k+m+1, l+n+1}$ by 


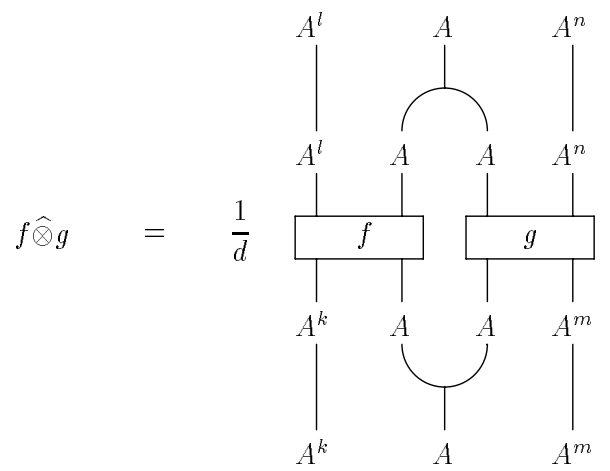

The following is easily checked by graphical computations.

\section{Lemma 3.3}

1. We have $f \widehat{\otimes} g \in D_{k+m, l+n}$.

2. The unit $1_{A}$ of $A_{0,0}$ satisfies $1_{A} \widehat{\otimes} f=f \widehat{\otimes} 1_{A}=f$ for $f \in D_{m, n}$.

3. For $f: V \otimes A \rightarrow W \otimes A, h: A \otimes X \rightarrow A \otimes Y$ and $g \in D_{m, n}$ with $m, n \geq 0$, we have $(f \widehat{\otimes} g) \widehat{\otimes} h=f \widehat{\otimes}(g \widehat{\otimes} h)$.

4. For $f \in D_{m, m^{\prime}}, f^{\prime} \in D_{m^{\prime}, m^{\prime \prime}}, g \in D_{n, n^{\prime}}$ and $g^{\prime} \in D_{n^{\prime}, n^{\prime \prime}}$, we have

$$
(f \widehat{\otimes} g)\left(f^{\prime} \widehat{\otimes} g^{\prime}\right)=\left(f f^{\prime}\right) \widehat{\otimes}\left(g g^{\prime}\right) .
$$

The block system $\left\{D_{m, n}\right\}$ is now a monoidal algebra by the previous lemma. The construction can be obviously extended to the systems $\left\{B_{m, n}\right\}$ and $\left\{C_{m, n}\right\}$ so that they give rise to a $2 \times 2$-bicategory $\mathcal{B}$ :

$$
\begin{gathered}
A_{m, m^{\prime}} \otimes B_{n, n^{\prime}} \subset B_{m+n, m^{\prime}+n^{\prime}}, \\
B_{m, m^{\prime}} \widehat{\otimes} D_{n, n^{\prime}} \subset B_{m+n, m^{\prime}+n^{\prime}}, \\
C_{m, m^{\prime}} \otimes A_{n, n^{\prime}} \subset C_{m+n, m^{\prime}+n^{\prime}}, \\
D_{m, m^{\prime}} \widehat{\otimes} C_{n, n^{\prime}} \subset C_{m+n, m^{\prime}+n^{\prime}}, \\
B_{m, m^{\prime}} \widehat{\otimes} C_{n, n^{\prime}} \subset A_{m+n-1, m^{\prime}+n^{\prime}-1}, \\
C_{m, m^{\prime}} \otimes B_{n, n^{\prime}} \subset D_{m+n-1, m^{\prime}+n^{\prime}-1}
\end{gathered}
$$

with analogous properties of tensor products for $\left\{D_{m, n}\right\}$.

If we denote by $H$ and $H^{*}$ objects associated to $B_{1,1}$ and $C_{1,1}$ respectively, then $A$ is identified with $H \otimes H^{*}$ and $D_{m, n}=\operatorname{Hom}\left(\left(H^{*} \otimes H\right)^{\otimes n},\left(H^{*} \otimes H\right)^{\otimes m}\right)$.

Proposition 3.4 The bicategory $\mathcal{B}$ is rigid. More precisely, the generators $H$ and $H^{*}$ are rigid with rigidity pairs given by

$$
\begin{gathered}
\delta: I \rightarrow H \otimes H^{*}=A, \quad T \in D_{0,1}=\operatorname{Hom}\left(H^{*} \otimes H, J\right), \\
S \in D_{1,0}=\operatorname{Hom}\left(J, H^{*} \otimes H\right), \quad \epsilon: H \otimes H^{*}=A \rightarrow I .
\end{gathered}
$$

( $J$ denotes the unit object for $D$.

Proof The hook identities for these pairs are nothing but the unit and counit identities for $T$ and $S$ respectively.

The rigidity pairs then induce the Frobenius algebra structure on $H^{*} \otimes H$ by switching the roles of $(\delta, \epsilon)$ and $(S, T)$, which is referred to as the dual Q-system: the multiplication and comultiplication in $H^{*} \otimes H$ are given respectively by

$1 \otimes \epsilon \otimes 1: H^{*} \otimes H \otimes H^{*} \otimes H \rightarrow H^{*} \otimes H, \quad 1 \otimes \delta \otimes 1: H^{*} \otimes H \rightarrow H^{*} \otimes H \otimes H^{*} \otimes H$. 
Now the following duality for algebraic Q-systems, although obvious, generalizes an operator algebraic result in [6].

Proposition 3.5 Given an algebraic Q-system $(S, T)$, its bidual Q-system is canonically isomorphic to $(S, T)$.

\section{Bicategory of Bimodules}

Recall that a morphism $f: X \rightarrow Y$ in a category is called a monomorphism (epimorphism respectively) if $g_{j}: Z \rightarrow X\left(g_{j}: Y \rightarrow Z\right)$ for $j=1,2$ satisfies $f g_{1}=f g_{2}\left(g_{1} f=g_{2} f\right)$, then $g_{1}=g_{2}$. A subobject of an object $Y$ is a pair $(X, j)$ of an object $X$ and a monomorphism $j: X \rightarrow Y$. A subobject $j: X \rightarrow Y$ is called a direct summand if we can find a morphism $p: Y \rightarrow X$ such that $p j=1_{X}$.

In what follows, categories are assumed to be linear, have splitting idempotents and be closed under taking direct sums. Given an idempotent $e \in \operatorname{End}(X)$, we denote the associated subobject of $X$ by $e X$ (with $e$ regarded as a monomorphism in $\operatorname{Hom}(e X, X)=\operatorname{End}(X) e)$, which is a direct summand of $X$ and we have the obvious identification $e X \oplus(1-e) X=X$.

Let $A$ be a Frobenius algebra in a tensor category $\mathcal{T}$. By a left $A$-module, we shall mean an object $M$ in $\mathcal{T}$ together with a morphism (called the action) $\lambda: A \otimes M \rightarrow M$ satisfying $\lambda\left(\epsilon \otimes 1_{M}\right)=1_{M}$ and the commutative diagram

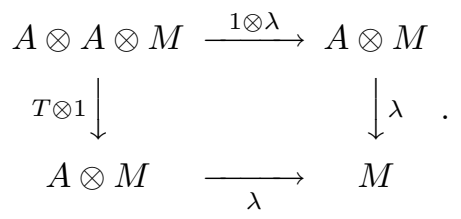

The notion of right $A$-module is defined analogously. Let $B$ be another Frobenius algebra. By an $A-B$ bimodule, we shall mean a left $A$-module $M$ (with the left action $\lambda: A \otimes M \rightarrow M$ ) which is a right $B$-module (with the right action $\mu: M \otimes B \rightarrow M)$ at the same time and makes the diagram

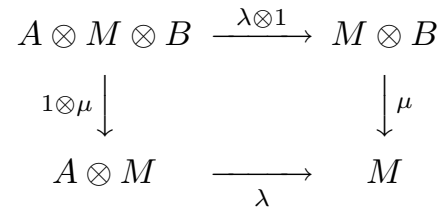

commutative.

An $A$ - $B$ bimodule based on an object $M$ in $\mathcal{T}$ is simply denoted by ${ }_{A} M_{B}$. Given another $A$ - $B$ bimodule ${ }_{A} N_{B}$, a morphism $f: M \rightarrow N$ in the category $\mathcal{T}$ is said to be $A-B$ linear if the diagram

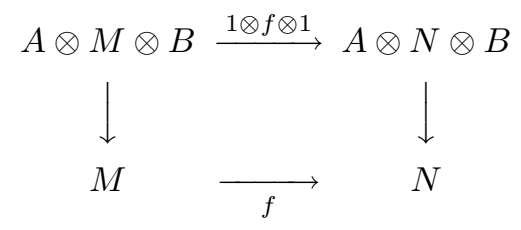

commutes.

The totality of $A-B$ bimodules $\left\{{ }_{A} M_{B}\right\}$ forms a linear category ${ }_{A} \mathcal{T}_{B}$ by

$$
\operatorname{Hom}\left({ }_{A} M_{B},{ }_{A} N_{B}\right)=\{f \in \operatorname{Hom}(M, N) ; f \text { is } A \text { - } B \text { linear }\} \text {. }
$$


Recall here that we have assumed splitting idempotents in the category $\mathcal{T}$ and the same holds for ${ }_{A} \mathcal{T}_{B}$ : if $e \in \operatorname{End}\left({ }_{A} M_{B}\right)$ is an idempotent, then the $A$ - $B$ action on $M$ induces an $A-B$ action on the subobject $e M$, i.e., ${ }_{A}(e M)_{B}$.

From here on we exclusively deal with Frobenius algebras of algebraic Qsystems, i.e., special Frobenius algebras, and shall introduce the notion of tensor product for bimodules. A more general and categorical construction is available in [3] but we prefer the following less formal description, which enables us to easily check the associativity (the so-called pentagonal relation) of tensor products.

Let $X_{B}$ and ${ }_{B} Y$ be right and left $B$-modules with action morphisms $\rho$ and $\lambda$ respectively. Let $e \in \operatorname{End}(X \otimes Y)$ be an idempotent defined by

$$
e=d^{-1}(\rho \otimes \lambda)\left(1_{X} \otimes \delta_{A} \otimes 1_{Y}\right),
$$

where $d$ is the common scalar for $T S$ and $\epsilon \delta$.

The module tensor product $X \otimes_{B} Y$ is, by definition, the subobject $e(X \otimes Y)$ of $X \otimes Y$ associated to the idempotent $e$. For bimodules ${ }_{A} X_{B}$ and ${ }_{B} Y_{C}, e$ belongs to $\operatorname{End}\left({ }_{A} X \otimes Y_{C}\right)$ and hence it induces an $A$-C bimodule ${ }_{A} X \otimes_{B} Y_{C}$.

Let ${ }_{C} Z$ be another left $C$-module and $f \in \operatorname{End}(Y \otimes Z)$ be the idempotent associated to the inner action of $C$. Then it is immediate to show the commutativity $\left(e \otimes 1_{Z}\right)\left(1_{X} \otimes f\right)=\left(1_{X} \otimes f\right)\left(e \otimes 1_{Z}\right)$ by the compatibility of left and right actions on $Y$, which enables us to identify

$$
\left(X \otimes_{B} Y\right) \otimes_{C} Z=\left(e \otimes 1_{Z}\right)\left(1_{X} \otimes f\right)(X \otimes Y \otimes Z)=X \otimes_{B}\left(Y \otimes_{C} Z\right) .
$$

Moreover, given morphisms $\varphi:{ }_{A} X_{B} \rightarrow{ }_{A} X_{B}^{\prime}$ and $\psi:{ }_{B} Y_{C} \rightarrow{ }_{B} Y_{C}^{\prime}, \varphi \otimes_{B} \psi:$ ${ }_{A} X \otimes_{B} Y_{C} \rightarrow{ }_{A} X^{\prime} \otimes_{B} Y_{C}^{\prime}$ is defined by

$$
\varphi \otimes_{B} \psi=(\varphi \otimes \psi) e=e^{\prime}(\varphi \otimes \psi),
$$

where $e^{\prime} \in \operatorname{End}\left(X^{\prime} \otimes Y^{\prime}\right)$ denotes the idempotent associated to the inner action of $B$ on $X^{\prime} \otimes Y^{\prime}$. It is also immediate to see the associativity for the tensor product of morphisms:

$$
\left(\phi \otimes_{A} \varphi\right) \otimes_{B} \psi=\phi \otimes_{A}\left(\varphi \otimes_{B} \psi\right) .
$$

(More precisely, the identification is through the natural isomorphisms among module tensor products of objects.)

The Frobenius algebra $A$ itself bears the structure of $A-A$ bimodule by the multiplication morphism, which is denoted by ${ }_{A} A_{A}$. Given a left $A$-module $\lambda$ : $A \otimes X \rightarrow X$, let $\lambda^{*}: X \rightarrow A \otimes X$ be the associated coaction: $\lambda^{*}=\left(1_{A} \otimes \lambda\right)\left(\delta_{A} \otimes 1_{X}\right)$.

Lemma 4.1 Both of $\lambda$ and $\lambda^{*}$ are A-linear.

Proof The $A$-linearity of $\lambda$ is just the associativity of the action. To see the $A$-linearity of $\lambda^{*}$, we use the identity

$$
\left(T \otimes 1_{A}\right)\left(1_{A} \otimes \delta_{A}\right)=S=\left(1_{A} \otimes T\right)\left(\delta_{A} \otimes 1_{A}\right) .
$$

Lemma 4.2 Let $e \in \operatorname{End}(A \otimes X)$ be the idempotent associated to the inner action of $A$ on $A \otimes X$. Then we have

$$
\lambda \lambda^{*}=d 1_{X}, \quad \lambda^{*} \lambda=d e .
$$

Proof These follow from simple graphical computations of $\lambda \lambda^{*}$ and $\lambda^{*} \lambda$. 
Lemma 4.3 The action morphism $\lambda: A \otimes X \rightarrow X$ induces the A-linear isomorphism $l: A \otimes_{A} X \rightarrow X$ with the inverse given by $d^{-1} \lambda^{*}$. Likewise a right $A$-module $\rho: X \otimes A \rightarrow X$ induces the isomorphism $r: X \otimes_{A} A \rightarrow X$ with the inverse given by $d^{-1} \rho^{*}$.

Here is another useful observation, which is an immediate consequence of definitions.

Lemma 4.4 Let $A$ be a Frobenius algebra. Then, by the correspondance $(\lambda$ : $A \otimes X \rightarrow X) \Longleftrightarrow\left(\lambda^{*}: X \rightarrow A \otimes X\right)$, there is an equivalence between the category of left $A$-modules and the category of left A-comodules.

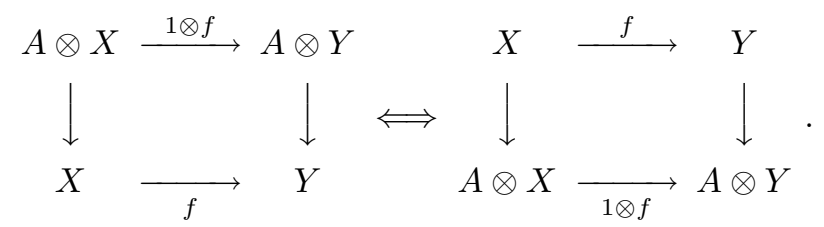

Lemma 4.5 Let $X_{A}$ be a right $A$-module and ${ }_{A} Y$ be a left $A$ module with the associated isomorphisms $r: X \otimes_{A} A \rightarrow X$ and $l: A \otimes_{A} Y \rightarrow Y$. Then $r$ and $l$ satisfy the triangle identity: $r \otimes_{A} 1_{Y}=1_{X} \otimes_{A} l$ on $X \otimes_{A} A \otimes_{A} Y$.

Proof Let $e_{X} \in \operatorname{End}(X \otimes A), e_{Y} \in \operatorname{End}(A \otimes Y)$ and $e \in \operatorname{End}(X \otimes Y)$ be idempotents associated to the inner actions of $A$. We need to show the equality

$$
e\left(\rho \otimes 1_{Y}\right)\left(e_{X} \otimes 1_{Y}\right)\left(1_{X} \otimes e_{Y}\right)=e\left(1_{X} \otimes \lambda\right)\left(e_{X} \otimes 1_{Y}\right)\left(1_{X} \otimes e_{Y}\right) .
$$

By a graphical computation, we see that

$d^{2}\left(\rho \otimes 1_{Y}\right)\left(e_{X} \otimes 1_{Y}\right)\left(1_{X} \otimes e_{Y}\right)=d(\rho \otimes \lambda)\left(1_{X} \otimes S \otimes 1_{Y}\right)=d^{2}\left(1_{X} \otimes \lambda\right)\left(e_{X} \otimes 1_{Y}\right)\left(1_{X} \otimes e_{Y}\right)$.

Summarizing the discussions so far, we have

Proposition 4.6 The family of categories $\left\{{ }_{A} \mathcal{T}_{B}\right\}$ indexed by pairs of special Frobenius algebras forms a bicategory with unit constraints given by $l$ and $r$ in the previous lemma.

The following is not needed in what follows but enables us to compare our definition with the one in [3].

Lemma 4.7 The projection $e: X \otimes Y \rightarrow X \otimes_{B} Y$ gives the cokernel of

$$
\left(\rho \otimes 1_{Y}-1_{X} \otimes \lambda\right): X \otimes B \otimes Y \rightarrow X \otimes Y .
$$

Proof By a graphical computation, we have

$$
e\left(\rho \otimes 1_{Y}\right)=(\rho \otimes \lambda)\left(1_{X} \otimes S \otimes 1_{Y}\right)=e\left(1_{X} \otimes \lambda\right) .
$$

Conversely, given a morphism $f: X \otimes Y \rightarrow Z$ satisfying $f\left(\rho \otimes 1_{Y}\right)=f\left(1_{X} \otimes \lambda\right)$, we can show $e f=f$. 


\section{Rigidity in Bimodules}

The rigidity of categorical modules is considered in [3] under the assumption of a certain 'commutativity' of Frobenius algebras. Although its general validity would be well-known for experts, we shall describe here the relevant points for completeness.

Let $A$ and $B$ be Frobenius algebras in a tensor category $\mathcal{T}$ and ${ }_{A} X_{B}$ be an $A$ - $B$ bimodule in $\mathcal{T}$. Assume that the object $X$ admits a (left) dual $X^{*}$ in $\mathcal{T}$ with a rigidity pair given by $\epsilon: X \otimes X^{*} \rightarrow I$ and $\delta: I \rightarrow X^{*} \otimes X$. We can then define the $B$ - $A$ action on $X^{*}$ as the transposed morphism: consider $B \otimes X^{*} \rightarrow X^{*}$ and $X^{*} \otimes A \rightarrow X^{*}$ defined by Fig. 2 .
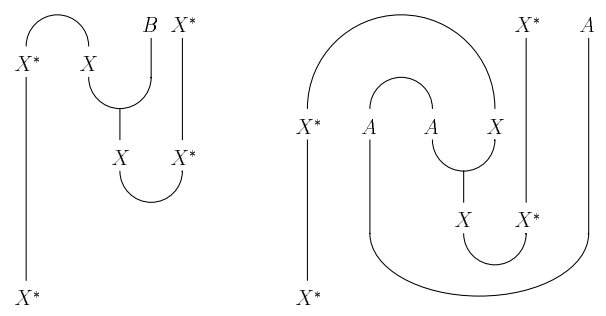

Figure 2

The following is immediate by easy graphical works.

Lemma 5.1 These in fact define the left and right actions on $X^{*}$, which are compatible in the following sense.
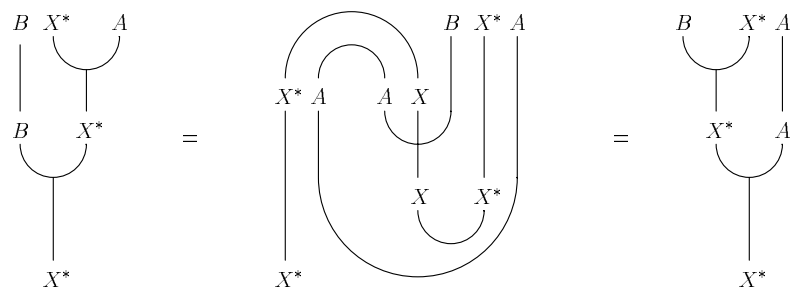

We shall show that the bimodule ${ }_{B} X_{A}^{*}$ is a dual object of ${ }_{A} X_{B}$. To this end, we first introduce morphisms $\epsilon: X \otimes X^{*} \rightarrow A$ and $\delta: B \rightarrow X^{*} \otimes X$ by Fig. 3
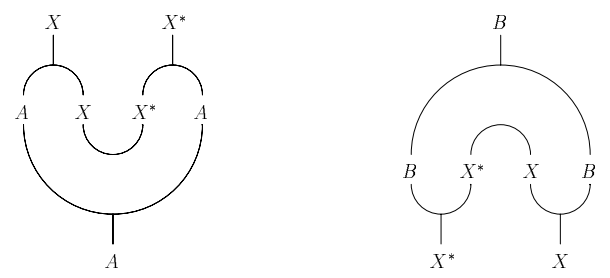

Figure 3

Lemma 5.2 We have

$$
\begin{aligned}
\epsilon & =d_{A}\left(1_{A} \otimes \epsilon_{X}\right)\left((X \rightarrow A \otimes X) \otimes 1_{X^{*}}\right), \\
\delta & =d_{B}\left(1_{X^{*}} \otimes(X \otimes B \rightarrow X)\right)\left(\delta_{X} \otimes 1_{B}\right) .
\end{aligned}
$$


Proof Insert the definition of (co)actions on $X^{*}$ and compute graphically.

Lemma 5.3 The morphism $\epsilon: X \otimes X^{*} \rightarrow A$ is $A$-A linear whereas the morphism $\delta: B \rightarrow X^{*} \otimes X$ is $B-B$ linear.

Proof We use the formulas in the above lemma together with the definition of (co)actions on $X^{*}$.

Lemma 5.4 Let $e_{B} \in \operatorname{End}\left(X \otimes X^{*}\right)$ and $e_{A} \in \operatorname{End}\left(X^{*} \otimes X\right)$ be idempotents associated with inner actions. Then $\epsilon$ and $\delta$ are supported by these idempotents: $\epsilon e_{B}=\epsilon$ and $e_{A} \delta=\delta$.

Proof For example, the equality $e_{A} \delta=\delta$ is proved by
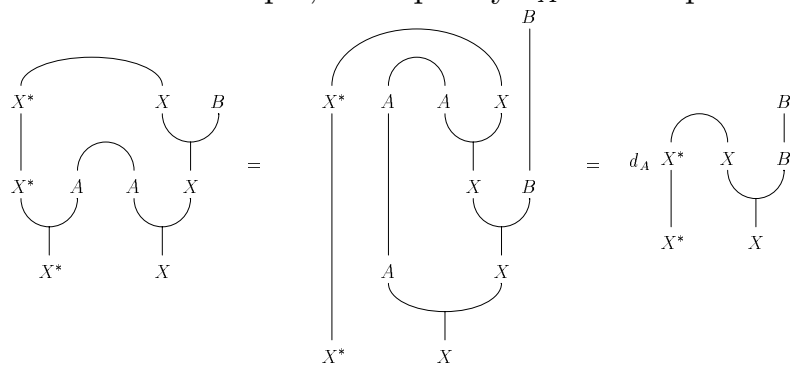

By the above lemmas, we can regard $\epsilon$ and $\delta$ as defining morphisms ${ }_{A} X \otimes_{B}$ $X_{A}^{*} \rightarrow{ }_{A} A_{A}$ and ${ }_{B} B_{B} \rightarrow{ }_{B} X^{*} \otimes_{A} X_{B}$ respectively.

Lemma 5.5 The compositions

$$
\begin{aligned}
& X \stackrel{1 \otimes_{B} \delta}{\longrightarrow} X \otimes_{B} X^{*} \otimes_{A} X \stackrel{\epsilon \otimes_{A} 1}{\longrightarrow} X, \\
& X^{*} \stackrel{\delta \otimes_{B} 1}{\longrightarrow} X^{*} \otimes_{A} X \otimes_{B} X^{*} \stackrel{1 \otimes_{A} \epsilon}{\longrightarrow} X^{*}
\end{aligned}
$$

are scalar multiplication of identities by the common scalar $d_{A}^{2} d_{B}$.

Proof By the previous lemma together with Corollary 4.3, we need to compare compositions

$$
\begin{gathered}
X \stackrel{d_{B}^{-1} \rho^{*}}{\longrightarrow} X \otimes B \stackrel{1_{X} \otimes \delta}{\longrightarrow} X \otimes X^{*} \otimes X \stackrel{\epsilon \otimes 1_{X}}{\longrightarrow} A \otimes X \stackrel{\lambda}{\longrightarrow} X, \\
X^{*} \stackrel{d_{B}^{-1} \lambda^{*}}{\longrightarrow} B \otimes X^{*} \stackrel{\delta \otimes 1_{X^{*}}}{\longrightarrow} X^{*} \otimes X \otimes X^{*} \stackrel{1_{X^{*}} \otimes \epsilon}{\longrightarrow} X^{*} \otimes A \stackrel{\rho}{\longrightarrow} X^{*},
\end{gathered}
$$

where $\lambda$ denotes one of the left actions $A \otimes X \rightarrow X, B \otimes X^{*} \rightarrow X^{*}$ and similarly for $\rho, \lambda^{*}$ and $\rho^{*}$.

By multiplying $d_{A}^{-1}$ on both of these compositions, the former is reduced to

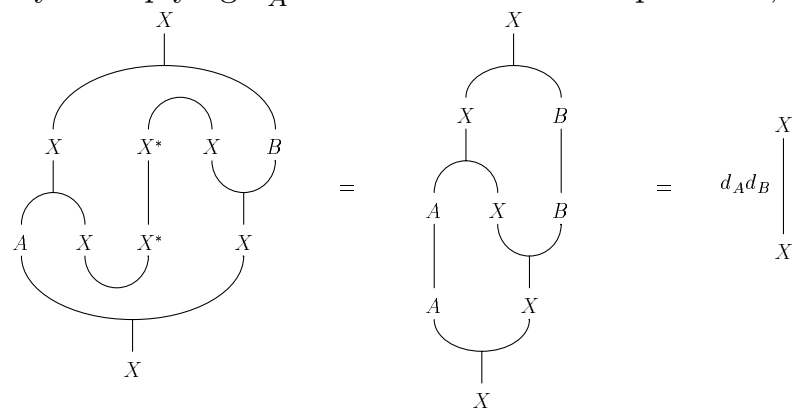


whereas the latter is given by

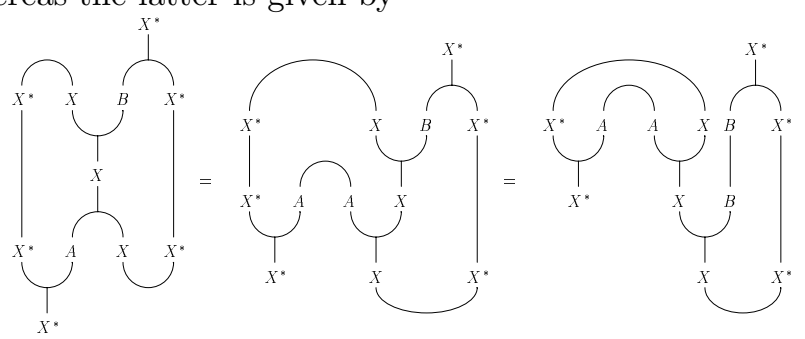

and turns out to be $d_{A} d_{B} 1_{X}$ from the relations in Fig. 4.
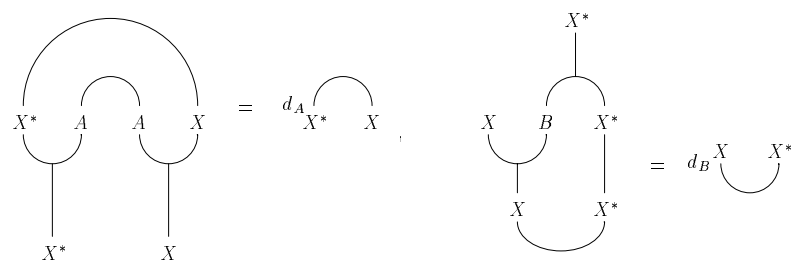

Figure 4

Proposition 5.6 Let ${ }_{A} X_{B}$ be a bimodule and assume that $X$ is rigid in $\mathcal{T}$. Then the bimodule ${ }_{A} X_{B}$ is rigid in the bicategory with the dual bimodule given by ${ }_{B} X_{A}^{*}$.

Proof This is just a paraphrase of the previous lemma.

Definition 5.7 Given a Frobenius algebra $A$ in a tensor category $\mathcal{T}$, we denote by ${ }_{A} \mathcal{T}_{A}$ the tensor category of $A$ - $A$ bimodules.

Proposition 5.8 Given a special Frobenius algebra $A$ in a tensor category $\mathcal{T}$, let $B$ be the dual Frobenius algebra of $A$. Then the bicategory connecting $A$ and $B$ is generated by the bimodule $H={ }_{I} A_{A}$ in $\mathcal{T}: H \otimes{ }_{A} H^{*} \cong A$ while the Frobenius algebra ${ }_{A} H^{*} \otimes H_{A}$ is isomorphic to $B$.

Theorem 5.9 (Duality for Tensor Categories) Given a special Frobenius algebra $A$ in a tensor category $\mathcal{T}$, the dual Frobenius algebra $B$ is canonically realized in the tensor category ${ }_{A} \mathcal{T}_{A}$ and the tensor category ${ }_{B}\left({ }_{A} \mathcal{T}_{A}\right)_{B}$ of $B$-B bimodules in ${ }_{A} \mathcal{T}_{A}$ is naturally monoidally equivalent to the starting tensor category $\mathcal{T}$.

Proof By the identification $B=H^{*} \otimes H$, the object $H$ has the structure of a right $B$-module in an obvious way and, if we regard this as defining an object $M$ at an off-diagonal corner of a bicategory connecting $\mathcal{T}$ and ${ }_{B}\left({ }_{A} \mathcal{T}_{A}\right)_{B}$, then it satisfies the imprimitivity condition; $M \otimes_{B} M^{*}=I$ (the unit object in $\mathcal{T}$ ) and $M^{*} \otimes M=$ ${ }_{B} B_{B}$ (the unit object in $\left.{ }_{B}\left({ }_{A} \mathcal{T}_{A}\right)_{B}\right)$. Thus taking adjoint tensor multiplications by $M$ gives rise to a monoidal equivalence of tensor categories in question. 
6 Semisimplicity

An object $X$ is said to be semisimple if any subobject is a direct summand and said to be simple if there is no non-trivial subobject.

Note that, if $\operatorname{End}(X)$ is finite-dimensional for a semisimple object $X$, then $X$ is isomorphic to a direct sum of simple objects.

A tensor category is semisimple if every object is semisimple.

The following is a direct and simplified version of the proof in $[3, \S 5.4]$ (cf. also $[10])$.

Proposition 6.1 Let $A$ and $B$ be special Frobenius algebras in a tensor category T. An $A$-B bimodule ${ }_{A} X_{B}$ is semisimple in ${ }_{A} \mathcal{T}_{B}$ if the base object $X$ is semisimple in $\mathcal{T}$.

Proof Let $f:{ }_{A} Y_{B} \rightarrow{ }_{A} X_{B}$ be a monomorphism in ${ }_{A} \mathcal{T}_{B}$. We first show that $f$ is monomorphic as a morphism in $\mathcal{T}$.

In fact, given a morphism $h: Z \rightarrow Y$ in $\mathcal{T}$ such that $f h=0$, the induced morphism $\widetilde{h}: A \otimes Z \otimes B \rightarrow Y$ defined by

$$
\widetilde{h}=(A \otimes Y \otimes B \rightarrow Y)\left(1_{A} \otimes h \otimes 1_{B}\right)
$$

is $A$ - $B$ linear and satisfies

$$
\widetilde{f}=(A \otimes Y \otimes B \rightarrow Y)\left(1_{A} \otimes f h \otimes 1_{B}\right)=0
$$

by the $A$ - $B$ linearity of $f$. Since $f$ is assumed to be monomorphic in ${ }_{A} \mathcal{T}_{B}$, this implies $\widetilde{h}=0$ and hence

$$
h=\widetilde{h}\left(\delta \otimes 1_{Z} \otimes \delta\right)=0,
$$

where $\delta$ denotes one of unit morphisms $I \rightarrow A$ and $I \rightarrow B$ in the Frobenius algebras.

So far, we have proved that $f: Y \rightarrow X$ gives a subobject of $X$. Since $X$ is semisimple by our assumption, we can find a morphism $g: X \rightarrow Y$ satisfying $g f=1_{Y}$. Let $\widetilde{g}: Y \rightarrow X$ be defined by

$$
\widetilde{g}=(A \otimes X \otimes B \rightarrow X)\left(1_{A} \otimes g \otimes 1_{B}\right)(Y \rightarrow A \otimes Y \otimes B),
$$

which is $A-B$ linear as a composition of $A-B$ linear morphisms.

Now the computation

$$
\begin{aligned}
\widetilde{g} f & =(A \otimes Y \otimes B \rightarrow Y)\left(1_{A} \otimes g \otimes 1_{B}\right)(Y \rightarrow A \otimes Y \otimes B) f \\
& =(A \otimes Y \otimes B \rightarrow Y)\left(1_{A} \otimes g f \otimes 1_{B}\right)(Y \rightarrow A \otimes Y \otimes B) \\
& =(A \otimes Y \otimes B \rightarrow Y)(Y \rightarrow A \otimes Y \otimes B) \\
& =d_{A} d_{B} 1_{Y}
\end{aligned}
$$

shows that ${ }_{A} Y_{B}$ is a direct summand of ${ }_{A} X_{B}$.

Corollary 6.2 Let $A$ and $B$ be special Frobenius algebras in a semisimpte tensor category $\mathcal{T}$. Then the category ${ }_{A} \mathcal{T}_{B}$ of $A-B$ bimodules in $\mathcal{T}$ is semisimple as well.

\section{Tannaka Duals}

By the Tannaka dual of a Hopf algebra $H$, we shall mean the tensor category of finite-dimensional (left) $H$-modules.

We shall here work with the Tannaka dual $\mathcal{A}$ of a semisimple Hopf algebra $H$ which is realized in a tensor category $\mathcal{T}$, i.e., we are given a faithful monoidal 
functor $F: \mathcal{A} \rightarrow \mathcal{T}$. The notion of $\mathcal{A}$-modules is introduced in [13] in terms of the notion of trivializing isomorphisms.

Let $\mathbb{A}$ be the unit object in the tensor category of $\mathcal{A}-\mathcal{A}$ modules in $\mathcal{T}$. Recall that the object $\mathbb{A}$ is isomorphic to

$$
\bigoplus_{V} F(V) \otimes V^{*}
$$

as an object in $\mathcal{T}$. By interchanging left and right actions, the dual object $\mathbb{A}^{*}$ of $\mathbb{A}$ is an $\mathcal{A}-\mathcal{A}$ module in a canonical way, which is isomorphic to the unit object $\mathcal{A}^{\mathbb{A}} \mathcal{A}$.

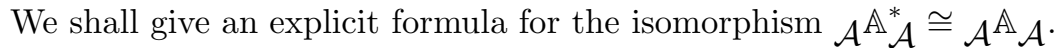

Lemma 7.1 The isomorphism $\mathbb{A}^{*} \rightarrow \mathbb{A}$ given by

$$
\mathbb{A}^{*}=\bigoplus_{V} F\left(V^{*}\right) \otimes V \stackrel{\oplus_{V} d(V) 1}{\longrightarrow} \bigoplus_{V} F\left(V^{*}\right) \otimes V=\mathbb{A}
$$

is $\mathcal{A}-\mathcal{A}$ linear.

Proof Let us prove the left $\mathcal{A}$-linearity for example. To this end, we first recall that the left action $F(U) \otimes \mathbb{A}^{*} \rightarrow \mathbb{A}^{*} \otimes U$ on $\mathbb{A}^{*}$ is given by the composition

$F(U) \otimes \mathbb{A}^{*} \rightarrow \mathbb{A}^{*} \otimes U \otimes U^{*} \otimes \mathbb{A} \otimes F(U) \otimes \mathbb{A}^{*} \rightarrow \mathbb{A}^{*} \otimes U \otimes \mathbb{A} \otimes F\left(U^{*}\right) \otimes F(U) \otimes \mathbb{A}^{*} \rightarrow \mathbb{A}^{*} \otimes U$.

We can check this formula by working on vector spaces: Let

$$
\left\{X \stackrel{\xi}{\longrightarrow} W \otimes U \stackrel{\xi^{*}}{\longrightarrow} X\right\}
$$

be an irreducible decomposition of $W \otimes U$ and $\left\{x_{l}\right\},\left\{w_{k}\right\},\left\{u_{i}\right\}$ be bases of vector spaces $X, W, U$ with the dual bases indicated by asterisk. Then

$$
\begin{aligned}
F(u) & \otimes F\left(v^{*}\right) \otimes v \\
& \mapsto \bigoplus_{W} \sum_{i, j, k} F\left(w_{j}^{*}\right) \otimes w_{k} \otimes u_{i} \otimes u_{i}^{*} \otimes F\left(w_{j}\right) \otimes w_{k}^{*} \otimes F(u) \otimes F\left(v^{*}\right) \otimes v \\
& \mapsto \bigoplus_{W} \sum_{i, j, k} \sum_{X, \xi, l} F\left(w_{j}^{*}\right) \otimes w_{k} \otimes u_{i} \otimes u_{i}^{*} \otimes F\left(x_{l}\right) \otimes \xi \otimes w_{k}^{*} \otimes F\left(v^{*}\right) \otimes v\left\langle x_{l}^{*}, \xi^{*}\left(w_{j} \otimes u\right)\right\rangle \\
& \mapsto \bigoplus_{W} \sum F\left(w_{j}^{*}\right) \otimes w_{k} \otimes u_{i} \otimes u_{i}^{*} \otimes F\left(x_{l}\right) \otimes \widetilde{\xi}\left(w_{k}^{*}\right) \otimes F\left(v^{*}\right) \otimes v\left\langle x_{l}^{*}, \xi^{*}\left(w_{j} \otimes u\right)\right\rangle
\end{aligned}
$$

$\left(F\left(x_{l}\right)\right.$ and $\widetilde{\xi}\left(w_{k}^{*}\right)$ being coupled with $F\left(v^{*}\right)$ and $u_{i}^{*} \otimes v$ respectively)

$$
\mapsto \bigoplus_{W} \sum F\left(w_{j}^{*}\right) \otimes w_{k} \otimes u_{i}\left\langle u_{i}^{*} \otimes v, \widetilde{\xi}\left(w_{k}^{*}\right)\right\rangle\left\langle v^{*}, \xi^{*}\left(w_{j} \otimes u\right)\right\rangle
$$

(letting $X=V$ )

$$
\begin{aligned}
& =\bigoplus_{W} \sum F\left(w_{j}^{*}\right) \otimes \xi v\left\langle v^{*}, \xi^{*}\left(w_{j} \otimes u\right)\right\rangle \\
& =\bigoplus_{W} \sum_{\xi} F\left(\widetilde{\xi^{*}}\left(u \otimes v^{*}\right)\right) \otimes \xi v \\
& =\bigoplus_{W} \sum_{\eta: U V^{*} \rightarrow W^{*}} \frac{d(V)}{d(W)} F\left(\eta\left(u \otimes v^{*}\right)\right) \otimes \widetilde{\eta^{*} v},
\end{aligned}
$$

where the family $\left\{W^{*} \stackrel{\eta^{*}}{\longrightarrow} U \otimes V^{*} \stackrel{\eta}{\longrightarrow} W^{*}\right\}$ denotes an irreducible decomposition of $U \otimes V^{*}$. 
Comparing the last expression with the definition of trivialization isomorphism $F(U) \otimes \mathbb{A} \rightarrow \mathbb{A} \otimes U$, we see that

$$
\mathbb{A}^{*}=\bigoplus_{V} F\left(V^{*}\right) \otimes V \stackrel{\oplus_{V} d(V) 1}{\longrightarrow} \bigoplus_{V} F\left(V^{*}\right) \otimes V=\mathbb{A}
$$

is $\mathcal{A}$-linear.

The object $A=\mathbb{A}^{*} \otimes \mathcal{A}^{\mathbb{A}}$ in $\mathcal{T}$ is a Frobenius algebra by the rigidity of $\mathcal{A}^{\mathbb{A}}$ : the multiplication morphism is given by

$$
A \otimes A=\mathbb{A}^{*} \otimes_{\mathcal{A}} \mathbb{A} \otimes \mathbb{A}^{*} \otimes_{\mathcal{A}} \mathbb{A} \stackrel{1 \otimes \epsilon \otimes 1}{\longrightarrow} \mathbb{A}^{*} \otimes_{\mathcal{A}} \mathbb{A} \otimes_{\mathcal{A}} \mathbb{A}=\mathbb{A}^{*} \otimes_{\mathcal{A}} \mathbb{A}=A .
$$

By the natural identification $\mathbb{A}^{*} \otimes \mathcal{A} \mathbb{A}=\mathbb{A}$, this can be rewritten as

$$
\mathbb{A} \otimes \mathbb{A} \rightarrow \mathbb{A} \otimes \mathbb{A}^{*} \stackrel{\epsilon}{\longrightarrow} \mathbb{A},
$$

where $\epsilon$ denotes a rigidity pair for $\mathcal{A}^{\mathbb{A}}(\epsilon$ being $\mathcal{A}-\mathcal{A}$ linear $)$ and is defined by the formula after Corollary 6.2:

$$
\begin{gathered}
\mathbb{A} \otimes \mathbb{A}^{*} \stackrel{\oplus_{X} d(X) 1 \otimes \epsilon_{X} \otimes 1}{\longrightarrow} \bigoplus_{X} \mathbb{A} \otimes X \otimes X^{*} \otimes \mathbb{A}^{*} \\
\longrightarrow \bigoplus_{X} F(X) \otimes \mathbb{A} \otimes \mathbb{A}^{*} \otimes X^{*} \stackrel{\oplus_{X} 1 \otimes \epsilon_{\mathbb{A}} \otimes 1}{\longrightarrow} \bigoplus_{X} F(X) \otimes X^{*}=\mathbb{A}
\end{gathered}
$$

with $\epsilon_{X}$ the ordinary vector space pairing and $\epsilon_{\mathbb{A}}$ the rigidity pairing for the object $\mathbb{A}$ in $\mathcal{T}$ (with the trivial action).

Since $\mathcal{A}_{\mathcal{A}}$ is identified with $\mathcal{A}^{\mathbb{A}_{\mathcal{A}}^{*}}$ by multiplying the weight $\left\{d(V)^{-1}\right\}_{V}$, the multiplication morphism $\mathbb{A} \otimes \mathbb{A} \rightarrow \mathbb{A}$ is given by the following process on vectors:

$$
\begin{aligned}
F(v) & \otimes v^{*} \otimes F(w) \otimes w^{*} \\
& \mapsto d(W)^{-1} F(v) \otimes v^{*} \otimes F(w) \otimes w^{*} \\
& \mapsto \bigoplus_{X} \frac{d(X)}{d(W)} \sum_{i} F(v) \otimes v^{*} \otimes x_{i} \otimes x_{i}^{*} \otimes F(w) \otimes w^{*} \\
& \mapsto \bigoplus_{X, U} \frac{d(X)}{d(W)} \sum_{\xi, i, j} F(v) \otimes \xi \otimes\left\langle u_{j}, \xi^{*}\left(v^{*} \otimes x_{i}\right)\right\rangle u_{j}^{*} \otimes x_{i}^{*} \otimes F(w) \otimes w^{*} \\
& \mapsto \bigoplus_{X, U} \frac{d(X)}{d(W)} \sum_{\xi, i, j} F(\widetilde{\xi} v) \otimes\left\langle u_{j}, \xi^{*}\left(v^{*} \otimes x_{i}\right)\right\rangle u_{j}^{*} \otimes x_{i}^{*} \otimes F(w) \otimes w^{*}
\end{aligned}
$$

(letting $U=W^{*}$ and $u_{j}=w_{j}^{*}$ for the pairing)

$$
\begin{aligned}
& \mapsto \bigoplus_{X} \frac{d(X)}{d(W)} \sum F\left(\langle\widetilde{\xi} v\rangle_{w}\right) \otimes\left\langle w_{j}^{*}, \xi^{*}\left(v^{*} \otimes x_{i}\right)\right\rangle\left\langle w_{j}, w^{*}\right\rangle x_{i}^{*} \\
& =\bigoplus_{X} \frac{d(X)}{d(W)} \sum_{\xi, i} F\left(\xi^{\prime}(v \otimes w)\right) \otimes\left\langle w^{*}, \xi^{*}\left(v^{*} \otimes x_{i}\right)\right\rangle x_{i}^{*} \\
& =\bigoplus_{X} \frac{d(X)}{d(W)} \sum_{\xi} F\left(\xi^{\prime}(v \otimes w)\right) \otimes{ }^{t}\left(\xi^{*}\right)^{\prime}\left(w^{*} \otimes v^{*}\right),
\end{aligned}
$$

where the family

$$
\left\{U^{*} \stackrel{\xi}{\longrightarrow} V^{*} \otimes X \stackrel{\xi^{*}}{\longrightarrow} U^{*}\right\}
$$

denotes an irreducible decomposition of $V^{*} \otimes X$ with $\widetilde{\xi}$ and $\xi^{\prime}$ Frobenius transforms of $\xi$. 
Since

$$
\frac{d(X)}{d(W)}\left(X \stackrel{\left(\xi^{*}\right)^{\prime}}{\longrightarrow} V \otimes W \stackrel{\xi^{\prime}}{\longrightarrow} X\right)
$$

gives an irreducible decomposition of $V \otimes W$, we have the following.

Proposition 7.2 The object $\mathbb{A}$ in $\mathcal{T}$ is an Frobenius algebra by the multiplication morphism

$$
F(v) \otimes v^{*} \otimes F(w) \otimes w^{*} \mapsto \bigoplus_{U} \sum_{\eta: U \rightarrow V W} F\left(\eta^{*}(v \otimes w)\right) \otimes{ }^{t} \eta\left(w^{*} \otimes v^{*}\right)
$$

(the family $\left\{U \stackrel{\eta}{\longrightarrow} V \otimes W \stackrel{\eta^{*}}{\longrightarrow} U\right\}$ being an irreducible decomposition of $V \otimes W)$ with the compatible rigidity copairing $\delta_{A}: \mathbb{A} \otimes \mathbb{A} \rightarrow I$ given by the composition

$$
\begin{aligned}
\bigoplus_{V, W} F(V) \otimes V^{*} \otimes F(W) \otimes W^{*} & \rightarrow \bigoplus_{V} F(V) \otimes V^{*} \otimes F\left(V^{*}\right) \otimes V \quad\left(\text { letting } W=V^{*}\right) \\
& \stackrel{\oplus_{V} d(V) 1}{\longrightarrow} \bigoplus_{V} F(V) \otimes V^{*} \otimes F\left(V^{*}\right) \otimes V \rightarrow I,
\end{aligned}
$$

where the last morphism is the summation of the canonical pairing

$$
F(V) \otimes F\left(V^{*}\right) \otimes V^{*} \otimes V \rightarrow I \otimes \mathbb{C}=I .
$$

The associated unit (morphism) is given by the obvious imbedding

$$
I \rightarrow F(\mathbb{C}) \otimes \mathbb{C} \subset \bigoplus_{V} F(V) \otimes V^{*} .
$$

Corollary 7.3 The multiplication morphism $\mathbb{A} \otimes \mathbb{A} \rightarrow \mathbb{A}$ is associative.

We have seen so far that Tannaka duals give rise to a special class of Frobenius algebras in a canonical way (a depth two characterization of the class is possible in terms of factorization of Frobenius algebras, see [13]). It is worth pointing out here that a similar computation is carried out in $[9, \S 6]$ based on the analysis of Hopf algebra strucutres. As can be recognized in the above arguments, our proof is purely categorical with the explicit use of fibre functors.

In what follows, we shall use calligraphic letters, say $\mathcal{A}$, to express Tannaka duals (realized in a tensor category $\mathcal{T}$ ) with the associated Frobenius algebras denoted by the corresponding boldface letters, say $\mathbb{A}$.

Recall here that Tannaka duals give rise to the bicategory of bimodules, whereas there is a natural notion of bimodule of Frobenius algebras which produces another bicategory.

We shall now construct a monoidal functor $\Phi$, which associate an $\mathbb{A}-\mathbb{B}$ bimodule to each $\mathcal{A}$ - $\mathcal{B}$ bimodule. For simplicity, consider a left $\mathcal{A}$-bimodule $X$ with the trivialization isomorphisms $\left\{\phi_{V}: F(V) \otimes X \rightarrow X \otimes V\right\}$. We choose a representative family $\left\{V_{j}\right\}$ of simple objects in the relevant Tannaka dual and set $\phi_{j}=\phi_{V_{j}}$.

The action morphism $\phi: \mathbb{A} \otimes X \rightarrow X$ is then introduced by

$$
\bigoplus_{j} \widetilde{\phi}_{j}: \bigoplus_{j} F\left(V_{j}\right) \otimes V_{j}^{*} \otimes X \rightarrow X
$$

where $\widetilde{\phi}_{j}: F\left(V_{j}\right) \otimes V_{j}^{*} \otimes X \rightarrow X$ corresponds to $\phi_{j}$ under the isomorphism $\operatorname{Hom}\left(F\left(V_{j}\right) \otimes V_{j}^{*} \otimes X, X\right) \cong \operatorname{Hom}\left(F\left(V_{j}\right) \otimes X, X\right) \otimes V_{j}=\operatorname{Hom}\left(F\left(V_{j}\right) \otimes X, X \otimes V_{j}\right)$. 
Now the square diagram

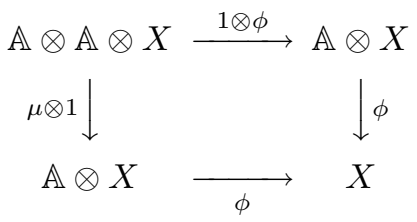

is commutative if and only if so is the diagram

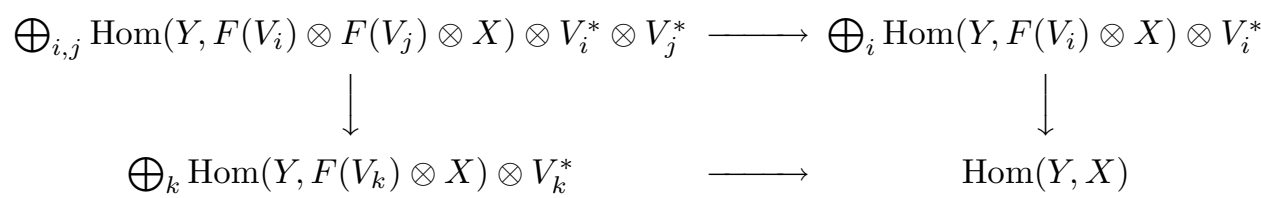

for any object $Y$. If we trace the morphisms starting from $f \otimes v_{i}^{*} \otimes v_{j}^{*}$ for $f: Y \rightarrow$ $F\left(V_{i}\right) \otimes F\left(V_{j}\right) \otimes X$ and $v_{i}^{*} \in V_{i}^{*}$, then the commutativity is reduced to the identity

$$
\sum_{k} \sum_{\xi}\left\langle\phi_{k}\left(\xi^{*} \otimes 1_{X}\right) f\right\rangle_{t} \xi\left(v_{i}^{*} \otimes v_{j}^{*}\right)=\left\langle\left(\phi_{i} \otimes 1\right)\left(1 \otimes \phi_{j}\right) f\right\rangle_{v_{i}^{*} \otimes v_{j}^{*}},
$$

where the family $\left\{V_{k} \stackrel{\xi}{\longrightarrow} V_{i} \otimes V_{j} \stackrel{\xi^{*}}{\longrightarrow} V_{k}\right\}$ denotes an irreducible decomposition.

Since the choice of $v_{i}^{*} \in V_{i}^{*}$ is arbitrary, the above relation is equivalent to

$$
\sum_{k, \xi}(1 \otimes \xi) \phi_{k}\left(\xi^{*} \otimes 1_{X}\right) f=\left(\phi_{i} \otimes 1\right)\left(1 \otimes \phi_{j}\right) f
$$

for any $f$ or simply

$$
\sum_{k, \xi}(1 \otimes \xi) \phi_{k}\left(\xi^{*} \otimes 1_{X}\right)=\left(\phi_{i} \otimes 1\right)\left(1 \otimes \phi_{j}\right),
$$

which is exactly the $\mathcal{A}$-module property of $X$, i.e., the commutativity of the diagram

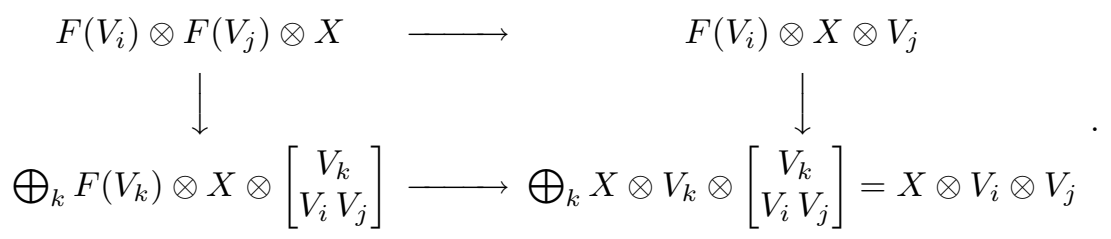

The unitality for the $\mathbb{A}$-action, which says that

$$
X=I \otimes X \rightarrow A \otimes X \rightarrow X
$$

is the identity, is reduced to that of the $\mathcal{A}$-action on $X$.

By summarizing the arguments so far, we have associated a left $\mathbb{A}$-module $\mathbb{A} X$ to each $\mathcal{A}$-module $\mathcal{A}^{X}$ with the common base object $X$ in $\mathcal{T}$. Moreover, given another $\mathcal{A}^{Y}$ with the associated $\mathbb{A} Y$, we have the equality

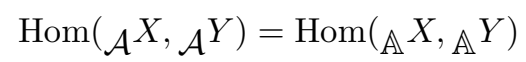

as subsets of $\operatorname{Hom}(X, Y)$ from our construction.

Thus the correspondance $\mathcal{A}^{X \mapsto \mathbb{A}} X$ defines a fully faithful functor $\Phi: \mathcal{A}^{\mathcal{T}} \rightarrow$ $\mathbb{A}^{\mathcal{T}}$.

We shall now identify the tensor products. Given a right $\mathcal{A}$-module $\mathcal{A}$ and a left $\mathcal{A}$-module $\mathcal{A}^{Y}$ in $\mathcal{T}$ with the trivialization isomorphisms $\phi_{V}: X \otimes F(V) \rightarrow V \otimes X$ 
and $\psi_{V}: F(V) \otimes Y \rightarrow Y \otimes V$, denote the associated action morphisms of $\mathbb{A}$ by $\phi: X \otimes \mathbb{A} \rightarrow X$ and $\psi: Y \otimes \mathbb{A} \rightarrow Y$ respectively.

Given a basis $\left\{v_{i}\right\}$ of $V$, we introduce morphisms $\phi_{V, i}: X \otimes F(V) \rightarrow X$ by the relation

$$
\phi_{V}=\sum_{i} v_{i} \otimes \phi_{V, i}
$$

in the vector space $\operatorname{Hom}(X \otimes F(V), V \otimes X)=V \otimes \operatorname{Hom}(F(V) \otimes X, X)$. Likewise, we define morphisms $\psi_{V, i}: F(V) \otimes Y \rightarrow Y$ so that

$$
\psi_{V}=\sum_{i} \psi_{V, i} \otimes v_{i}
$$

From the definition of $\phi, \phi \otimes 1_{Y}$ is identified with

$$
\bigoplus_{V} \sum_{i} v_{i} \otimes \phi_{V, i} \quad \in \bigoplus_{V} V \otimes \operatorname{Hom}(X \otimes F(V) \otimes Y, X \otimes Y)
$$

in the vector space

$$
\begin{aligned}
\operatorname{Hom}(X \otimes \mathbb{A} \otimes Y, X \otimes Y) & =\bigoplus_{V} \operatorname{Hom}\left(X \otimes F(V) \otimes V^{*} \otimes Y, X \otimes Y\right) \\
& =\bigoplus_{V} V \otimes \operatorname{Hom}(X \otimes F(V) \otimes Y, X \otimes Y) .
\end{aligned}
$$

Similarly we have the expression

$$
1_{X} \otimes \psi=\bigoplus_{V} \sum_{i} v_{i} \otimes \psi_{V, i} \quad \text { in } \bigoplus_{V} V \otimes \operatorname{Hom}(X \otimes F(V) \otimes Y, X \otimes Y) .
$$

Now the idempotent $p \in \operatorname{End}(X \otimes Y)$ producing the relative tensor product $X \otimes_{\mathbb{A}} Y$ is given by

$$
\sum_{V} \frac{d_{F(V)}}{d_{\mathbb{A}}} \sum_{i}\left(\phi_{V, i} \otimes 1_{Y}\right)\left(1_{X} \otimes \psi_{V, i}^{*}\right)
$$

from the definition of $p$ and the formula for $\delta_{A}$. Here we denote by $\left\{\psi_{V, i}^{*}: Y \rightarrow\right.$ $F(V) \otimes Y\}$ the cosystem of $\left\{\psi_{V, i}\right\}_{i}$ :

$$
\psi_{V, i} \psi_{V, j}^{*}=\delta_{i j} 1_{Y}, \quad \sum_{i} \psi_{V, i}^{*} \psi_{V, i}=1_{F(V) \otimes Y}
$$

and $d_{F(V)}, d_{\mathbb{A}}$ are quantum dimensions of the objects $F(V), \mathbb{A}$ respectively.

We next derive an explicit formula for the idempotent $\pi(e)$ which is used to define $X \otimes_{\mathcal{A}} Y$. Recall here that $\pi$ is an algebra homomorphism of the dual Hopf algebra $H^{*}$ into $\operatorname{End}(X \otimes Y)$ and $e \in H^{*}$ denotes the counit functional of $H$.

By using the explicit definition of $\pi$ in [13], we see that

$$
\pi(e)=\sum_{V} \frac{\operatorname{dim}(V)}{\operatorname{dim}(H)} \sum_{i}\left(\phi_{V, i} \otimes 1_{Y}\right)\left(1_{X} \otimes \psi_{V, i}^{*}\right),
$$

which is exactly the idempotent $p$ because of $d_{F(V)}=\operatorname{dim}(V)$ and $d_{\mathbb{A}}=\operatorname{dim}(H)$.

Proposition 7.4 The fully faithful functor $\Phi: \mathcal{A}^{\mathcal{T}_{\mathcal{B}}} \rightarrow \mathbb{A}^{\mathcal{T}_{\mathbb{A}}}$ is monoidal by the equality $X \otimes_{\mathcal{B}} Y=X \otimes_{\mathbb{B}} Y$ in $\mathcal{T}$.

Proposition 7.5 The monoidal functor $\Phi: \mathcal{A}_{\mathcal{B}} \rightarrow \mathbb{A}_{\mathbb{B}}$ is an equivalence of categories, i.e., any $\mathbb{A}-\mathbb{B}$ bimodule in $\mathcal{T}$ is isomorphic to $\Phi\left(\mathcal{A}^{X_{\mathcal{B}}}\right)$ with $\mathcal{A}^{X_{\mathcal{B}}}$ an $\mathcal{A}-\mathcal{B}$ bimodule in $\mathcal{T}$. 
Proof Let $\mathbb{A} X_{\mathbb{B}}$ be an $\mathbb{A}-\mathbb{B}$ bimodule in $\mathcal{T}$. Since the $\mathbb{A}-\mathbb{B}$ bimodule $\mathbb{A} \otimes X \otimes \mathbb{B}_{\mathbb{B}}$

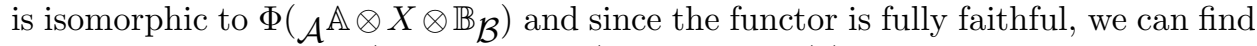
an idempotent $p \in \operatorname{End}\left(\mathcal{A} \mathbb{A} \otimes X \otimes \mathbb{B}_{\mathcal{B}}\right)$ such that $\Phi(p)$ induces the relative tensor

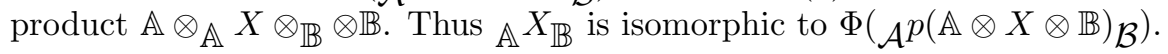

\section{References}

[1] P. Deligne, Catégories tannakiennes, 111-195, The Grothendieck Festshrift, II (Progress in Math. 87), Birkhäuser, 1990.

[2] S. Doplicher and J.E. Roberts, A new duality theory for compact groups, Invent. Math., 98(1989), 157-218.

[3] J. Fuchs and C. Schweigert, Category theory for conformal boundary conditions, Fields Institute Commun., to appear.

[4] D. Kazhdan and H. Wenzl, Reconstructing monoidal categories, Adv. Soviet Math., 16(1993), $111-136$.

[5] F.W. Lawvere, Ordinal sums and equational doctrines. Sem. on Triples and Categorical Homology Theory (ETH, Zürich, 1966/67). pp. 141-155. Springer, Berlin (1969).

[6] R. Longo, A duality for Hopf algebras and for subfactors. I, Commun. Math. Phys., 159(1994), $133-150$.

[7] R. Longo and J.E. Roberts, A theory of dimension, K-Theory, 11(1997), 103-159.

[8] S. Mac Lane, Categories for the working mathematician, 2nd ed., Springer Verlag, 1998.

[9] M. Müger, From subfactors to categories and topology I., J. Pure and Applied Algebras, $180(2003), 81-157$.

[10] V. Ostrik, Module categories, weak Hopf algebras and modular invariants, Transf. Groups, to appear.

[11] D. Tambara, A duality for modules over monoidal categories of representations of semisimple Hopf algebras, J. Algebra, 241(2001), 515-547.

[12] S. Yamagami, Group symmetry in tensor categories and duality for orbifolds, J. Pure and Applied Algebras, 167(2002), 83-128.

[13] _ Tannaka duals in semisimple tensor categories, J. Algebra, 253(2002), 350-391. 